\title{
ANÁLISE DA EFICIÊNCIA DE JOGADORES DOS PRINCIPAIS CAMPEONATOS EUROPEUS DE FUTEBOL ATRAVÉS DE DEA ${ }^{1}$
}

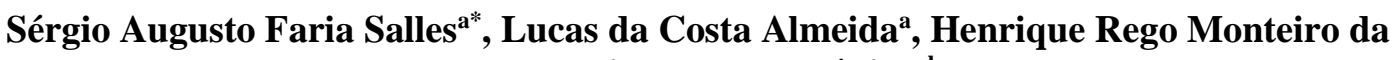 \\ Hora $^{\text {a }}$, Milton Erthal Júnior ${ }^{\text {ab }}$

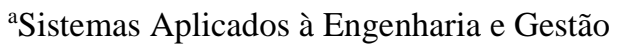 \\ Instituto Federal Fluminense - IFF, Campos dos Goytacazes-RJ, Brasil

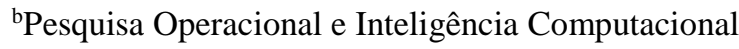 \\ Universidade Candido Mendes - UCAM, Campos dos Goytacazes-RJ, Brasil
}

Recebido 04/09/2017, aceito 30/11/2017

\begin{abstract}
RESUMO
Esta pesquisa objetiva analisar a eficiência de jogadores de sete campeonatos europeus de futebol, quanto a suas respectivas posições, e testar as seguintes hipóteses: Quanto maior o valor de mercado, mais eficiente será o jogador ("H1") e jogadores são mais eficientes na fase intermediária de suas carreiras ("H2"). Como método foi utilizada a Análise Envoltória de Dados, assumindo o modelo de retorno constante de escala (CCR). Foram analisadas 1.799 DMU's, três inputs e 26 outputs, tendo os resultados apontado a eficiência de $49 \%$ das alternativas. Para teste das hipóteses foi calculada a correlação entre variáveis na forma Pearson. A hipótese "H1" foi testada e a correlação resultou em "fraca" para duas das oito posições, enquanto o restante resultou em "muito fraca". Para a hipótese "H2" não foi possível identificar nenhum tipo de padrão nas relações entre as variáveis e os jogadores eficientes.
\end{abstract}

Palavras-chave: Eficiência, Futebol, DEA.

\begin{abstract}
This research aims to analyze the efficiency of players of seven European football championships, in relation to their respective positions, and test the following hypotheses: The higher the market value, the more efficient the player is ("H1") and the players are more efficient in the intermediate phase of their careers ("H2"). Data Envelopment Analysis was used as a method, assuming the constant scale return (CCR) model. 1799 DMU's, three inputs and 26 outputs were analyzed, where the results indicated the efficiency of $49 \%$ of the alternatives. To test the hypotheses, the correlation between variables in the Pearson form was calculated. Hypothesis "H1" was tested and the correlation resulted in "weak" for two of the eight positions, while the remainder resulted in "very weak". For the hypothesis "H2" it was not possible to identify any type of pattern in the relations between the variables and the efficient players.
\end{abstract}

Keywords: Efficiency, Soccer, DEA.

*Autor para correspondência. E-mail: safsalles@ hotmail.com.

DOI: 10.4322/PODes.2018.002 


\section{Introdução}

Durante o Século 20, ficou evidente que o esporte foi instituído como um fenômeno cultural, causando um grande impacto social, econômico e político. Atualmente, o futebol se apresenta como um dos meios de expressão mais importantes do esporte, além de ser um negócio de extrema importância econômica. Porém, nas duas últimas décadas, a indústria do futebol mudou significativamente, fazendo com que a sobrevivência econômica ganhasse um destaque cada vez maior (Zambom-Ferraresi et al., 2017). Além disto, González-Gómez e Picazo-Tadeo (2010) afirmam que, em vários países, o futebol é o esporte mais popular, tendo na Europa e América do Sul o maior número de apreciadores. Devido ao número crescente de pessoas que frequentam os jogos em estádios, aos fãs que possui e à renda que é gerada, é notório que o futebol gera satisfação e bem-estar nas pessoas.

Pestana Barros et al. (2010) argumentam que, indiretamente, a eficiência se relaciona com os resultados esportivos, de forma que as áreas operacionais e financeiras são impulsionadas a partir do sucesso que o clube obtém. Os autores ainda destacam a importância da eficiência, já que ela apresenta uma medida do desempenho, permitindo identificar mais precisamente pontos que necessitam ser corrigidos, ou revistos. Ademais, a utilização da eficiência auxilia na preparação de um ambiente favorável ao benchmarking entre os clubes concorrentes. Oukil e Govindaluri (2017) alegam que o ranqueamento de jogadores sempre foi relevante para auxiliar na tomada de decisões-chave na esfera do futebol.

A Análise Envoltória de Dados, ou DEA (Data Envelopment Analysis), é um método não paramétrico na pesquisa de operações, utilizado para calcular a eficiência relativa de instituições que sejam comparáveis e também para apoiar um benchmarking na gestão de operações (ElDemerdash et al., 2016). As instituições, ou entidades comparáveis, são chamadas de Decision Making Units (DMUs), transformando inúmeros inputs em vários outputs. O objetivo de DEA é avaliar a eficiência de cada DMU em relação aos seus pares, resultando em DMUs classificadas como eficientes ou ineficientes e também apontando a causa da ineficiência, se houver, e o nível necessário para corrigir o desempenho e melhorá-lo (El-Demerdash et al., 2013).

Neste contexto, este trabalho objetiva analisar a eficiência, através do método de Análise Envoltória de Dados, de jogadores dos campeonatos alemão, inglês, espanhol, francês, holandês, italiano e turco de acordo com a posição de cada um desses jogadores, medindo e comparando o desempenho de diferentes jogadores, em diferentes ligas. Posteriormente, serão testadas as seguintes hipóteses: quanto maior for o valor de mercado, mais eficiente será o jogador; jogadores são mais eficientes na fase intermediária de suas carreiras. Este trabalho se destaca por ser um dos poucos que analisa individualmente cada jogador, ao invés de analisar clubes de futebol. O trabalho recente de Oukil e Govindaluri (2017), por exemplo, analisa o desempenho individual dos jogadores, porém somente com base nos clubes de futebol da European Premier League Football (campeonato inglês), ou seja, não faz uma análise com uma base de dados extensa em comparação à do presente trabalho.

\section{Análise Envoltória de Dados e Futebol}

Os negócios no meio futebolístico cresceram de forma significativa nos últimos 20 anos, fazendo com que os clubes se tornassem grandes empresas e que é necessário que haja a melhoria da eficiência para que o negócio seja lucrativo e bem-sucedido no campo (Pyatunin et al., 2016). Ribeiro e Lima (2012) comentam que os clubes gastam dinheiro de diferentes formas em seus jogadores e usufruem de vários meios de incentivo para alcançar os objetivos em cada temporada. Além disso, é crucial para os gestores de clubes de futebol selecionar os melhores jogadores, estando isso relacionado aos custos e desempenho da equipe (Arabzad et al., 2013). Ademais, Espitia-Escuer e García-Cebrián (2016) comentam que os clubes de futebol sendo consideradas organizações econômicas, devem atingir seus objetivos sem consumir recursos demasiadamente, ou seja, devem ser eficientes. Guzmán e Morrow (2007) afirmam que os clubes de futebol são empresas incomuns, pois seu desempenho é julgado dentro e fora do campo. Kiani Mavi et al. (2012) discorrem que os gestores buscam métodos aprimorados que 
possam medir o desempenho de suas organizações devido ao fato de possuírem um compromisso na melhoria de suas unidades operacionais. De acordo com Villa e Lozano (2016), a Análise Envoltória de Dados tem sido amplamente utilizada para avaliar a eficiência em vários tipos de esporte, especialmente no futebol.

Zambom-Ferraresi et al. (2017) argumentam que uma simples observação do resultado é a maneira mais comum para avaliar o desempenho no esporte, sendo esta uma avaliação realizada a partir da perspectiva da eficácia. Porém é realmente necessário e importante a avaliação pela perspectiva da eficiência, assim como é para a grande parte das organizações. Haas (2003) já dizia sobre a eficiência produtiva em clubes de futebol, na qual a transformação de insumos em resultados é um processo de produção.

A DEA permite uma avaliação da eficiência considerando questões esportivas e financeiras ao mesmo tempo, sem assumir qualquer pressuposto sobre a função objetiva de um gestor de clube. O modelo foi proposto incialmente por Charnes et al. (1978) e agrega características de múltiplos resultados de um problema. O modelo é flexível, fazendo com que isto seja uma qualidade e também uma limitação, pois permitir que o modelo pondere os pesos na maximização pode levar a casos extremos (Jardin, 2009).

El-Demerdash et al. (2013) alegam que a DEA calcula o score de eficiência para diversas DMUs, sendo que o modelo depende da maximização de uma função de produção estimada pela DEA. Assim, para quaisquer inputs, o valor desta estimativa DEA indica o rendimento máximo de acordo com os inputs em todos os aspectos. Em contrapartida, para quaisquer outputs, o valor da estimativa DEA define o input mínimo que produz um determinado output em todos os aspectos. Assim, compara-se à fronteira paramétrica com desvios unilaterais estimados com o uso de métodos de programação matemática.

Oukil e Govindaluri (2017) afirmam que o ranking dos jogadores sempre foi uma contribuição importante no auxílio à tomada de decisões-chaves no meio futebolístico. Neste sentido, Adler et al. (2002) destacaram o uso de DEA como ferramenta para rankeamento, nas mais variadas áreas, classificando os métodos de rankeamento propostos na literatura em seis áreas, ou técnicas, básicas: técnica de eficiência cruzada e super eficiência, avaliação por benchmarking, rankeamento com estatísticas multivariadas e por DMUs ineficientes.

Petrović Djordjević et al. (2015) diz que os valores reais obtidos das partidas de futebol devem ser levados em consideração pelos modelos de Análise Envoltória de Dados (DEA), a fim de identificar os pontos fracos de um time de futebol, com base nas táticas de ataque e defesa. $\mathrm{O}$ autor ainda exemplifica, comentando que na primeira fase, por exemplo, o talento do jogador pode ser utilizado para dois outputs, um positivo para gols marcados e outros negativos para gols recebidos, sendo estes que devem ser minimizados. Esses são os indicadores de input de ataque e defesa, podendo ter valores negativos ou positivos, levando a uma estimativa da eficiência da produção de ataques, independentemente da defesa. Considerando o ataque, algumas ações por parte dos jogadores para aumentar as chances de marcar gols são: posse de bola e movimentação por passes, drible, chutes a gol, entre outros. Esses três indicadores supracitados podem ser variáveis de inputs, no qual contém o tempo que o time possuiu a bola, apontando o nível de ataque do time. Já para defesa, as ações defensivas observadas são como tirar a bola do time adversário para então realizar um novo ataque, como por exemplo a recuperação da posse de bola. Os inputs de defesa podem, sem obrigatoriedade, ser apresentados pelo tempo no qual o time adversário possuiu a bola, apontando o nível de defesa da equipe.

A DEA pode ser utilizado em conjunto com outras técnicas, como é o caso do trabalho de Rubem e Brandão (2015) que faz o uso da combinação de DEA com programação linear objetiva múltipla, e o trabalho de $\mathrm{Hu}$ et al. (2015) que utiliza a DEA e também PageRank. Complementando, outros métodos decisórios podem ser utilizados para classificação, como é o caso de Dadelo et al. (2014) que sugerem a utilização do método TOPSIS a fim de classificar equipes e jogadores, sendo também uma alternativa. Quanto a aquisição de informações pela técnica, a pesquisa de Sexton et al. (1986) contribui com uma extensa revisão sobre outras ferramentas que podem ser utilizadas em conjunto com a DEA, tais como a programação por objetivo e a análise de clusters. 


\section{Metodologia}

Segundo Gil (2010), a presente pesquisa possui caráter exploratório, visto que busca construir e confirmar hipóteses sobre o assunto estudado. Se configura como ex-post facto, analisando dados anteriores a sua elaboração, a fim de identificar e analisar relações entre variáveis. Por utilizar ferramentas estatísticas e realizar sua análise com base em dados numéricos, possui abordagem quantitativa. Sua natureza é aplicada, já que objetiva gerar conhecimentos direcionados a seu objeto de estudo (Silva e Menezes, 2005).

\subsection{Análise Envoltória de Dados}

Ruggiero (2011) define a Análise Envoltória de Dados como uma técnica da Pesquisa Operacional que objetiva mensurar a eficiência das chamadas unidades tomadoras de decisão, ou decision making units (DMU), tendo suas variáveis tratadas como inputs e outputs, assumindo o modelo de retorno constante (CCR), ou variável (BCC), de escala.

Na presente pesquisa, será utilizado o modelo CCR, proposto por Charnes et al. (1978). Este modelo trabalha com retornos constantes de escala, fazendo com que cada variação nos inputs altere, proporcionalmente, os outputs. A escolha pelo modelo deu-se, por sua capacidade de realizar uma maior diferenciação entre os resultados de eficiência obtidos. O cálculo do modelo DEA CCR é realizado de acordo com a Equação 1 (input) e 2 (output), estruturadas conforme Périco et al. (2008).

$$
\begin{aligned}
& \operatorname{Max} h_{O} \sum_{r=1}^{s} u_{r} y_{r o} \\
& \text { s.a. } \\
& \sum_{r=1}^{m} v_{i} x_{i k}=1 \\
& \sum_{r=1}^{n} u_{r} y_{r j}-\sum_{i=1}^{n} v_{i} x_{i j} \leq 0 \\
& u_{r}, v_{i} \geq 0
\end{aligned}
$$

$$
\begin{aligned}
& \operatorname{Min} h_{O} \sum_{i=1}^{n} v_{i} x_{i k} \\
& \text { s.a. } \\
& \sum_{r=1}^{m} u_{r} y_{r k}=1 \\
& \sum_{r=1}^{m} u_{r} y_{r j}-\sum_{i=1}^{n} v_{i} x_{i j} \leq 0 \\
& u_{r}, v_{i} \geq 0
\end{aligned}
$$

Sendo que:

- $x_{\mathrm{i}}=$ Inputs;

- $y i=$ Outputs;

- $k=D M U$

- ve u=Pesos para inputs e outputs;

- $h o=D M U$ da função objetivo. 
São consideradas eficientes as DMU's que obtiverem o resultado igual a 1, ou seja, $100 \%$ eficientes. Estas estarão inseridas na fronteira de eficiência do modelo. O modelo pode ainda ter duas orientações: De acordo com as entradas (inputs), ou saídas (outputs). As variáveis são as mesmas em ambas as orientações, a única alteração é que na orientação escolhida serão maximizados seus valores relacionados, enquanto o outro permanecerá inalterado.

O software utilizado para cálculo das eficiências das DMU's e procedimentos de DEA foi o MaxDEA 7 Basic, disponibilizado gratuitamente em: http://maxdea.com/MaxDEA.htm.

\subsection{Coleta de Dados e Procedimentos Técnicos}

A presente pesquisa utilizou, como medida de desempenho, estatísticas e informações de jogadores e clubes participantes dos campeonatos nacionais da Alemanha, Espanha, França, Holanda, Inglaterra, Itália e Turquia, referentes à temporada 2015/2016. Tais campeonatos foram escolhidos por seus dados estarem presentes em todas as bases consultadas, além de estarem ranqueadas entre as 11 maiores competições nacionais de clubes (UEFA, 2016). Os dados utilizados para construção do modelo foram extraídos de três websites: WhoScored (2016), Squawka (2016) e Transfermarkt (2016).

A avaliação de eficiência deu-se por posição de cada jogador, já que cada uma destas posições possui obrigações diferentes, sendo variantes de acordo com esquema tático do time no qual se insere o jogador. A Figura 1 (A) representa a divisão de posições conforme o site WhoScored. Embora cada jogador possa atuar em mais de uma posição durante uma competição (ou durante uma partida), esta pesquisa dividiu-os de acordo com a posição nas quais foram escalados (inicialmente) na maioria de seus jogos. Tal divisão resultou em 16 posições, sendo que foram consideradas diferentes as posições com mesmas funções, mas atuantes em diferentes lados do campo (esquerdo e direito). Considerando a similaridade de algumas destas funções, ilustrada pela Figura 1 (B), os autores optaram por agrupá-las, reduzindo para oito o número de posições, sendo criadas novas denominações para os conjuntos, conforme Figura 1 (C).

Portanto, esta pesquisa realizará a avaliação de eficiência de jogadores agrupados de acordo com oito posições: Goleiros, Zagueiros, Laterais, Meias (Atacantes, Centrais e Laterais), Pontas e Atacantes.

Figura 1: Representação das posições de jogadores em campo de futebol. G: Goleiro; Z: Zagueiro; L: Lateral (E.: Esquerdo, D.: Direito); V: Volante. A: Ala (E.: Esquerdo, D.: Direito); M: Meio Campo (E.: Esquerdo, D.: Direito); MC: Meia central; ML: Meia Lateral; MA: Meia

Atacante (E.: Esquerdo, D.: Direito); P: Ponta. AT: Atacante (E.: Esquerdo, D.: Direito).

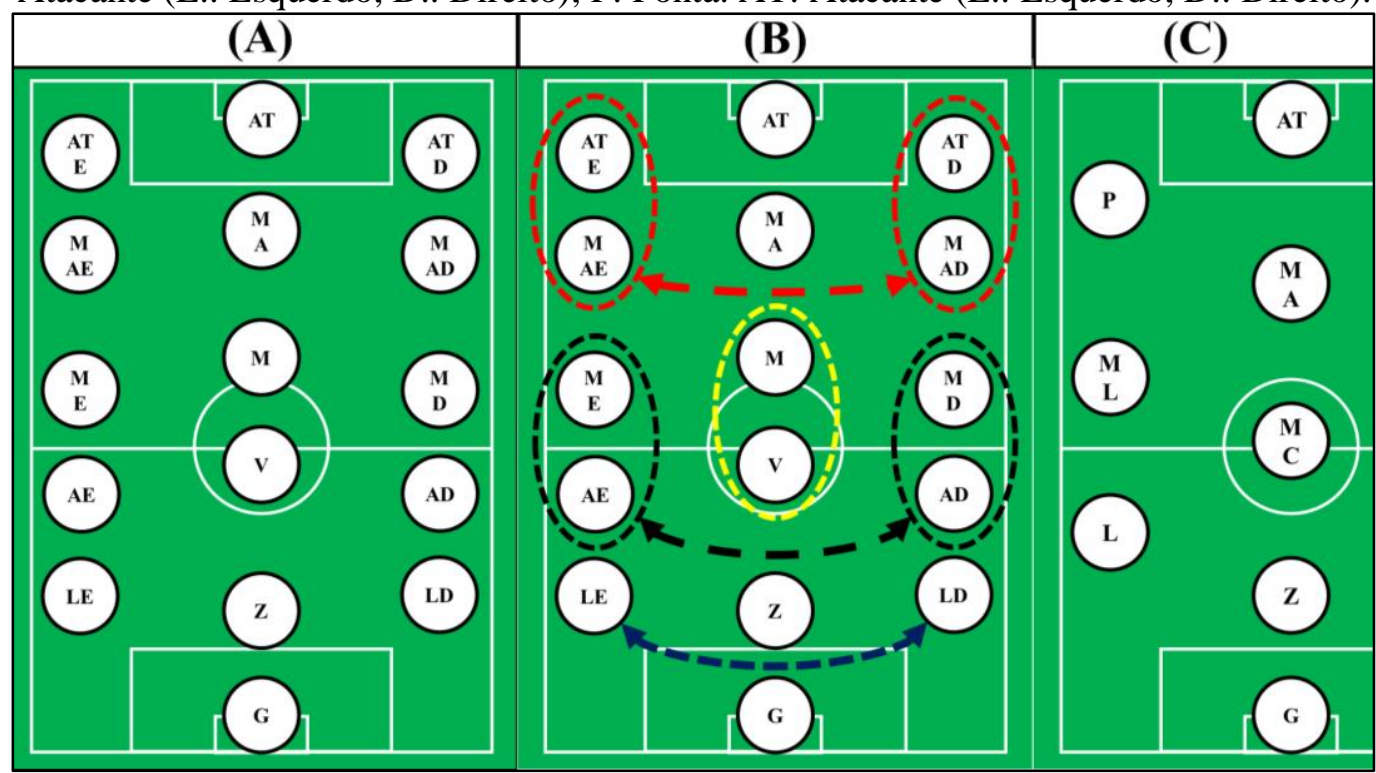

Fonte: Adaptado de WhoScored (2016). 
Ao final da coleta de dados haviam um total de três variáveis designadas como inputs e 96 variáveis como outputs. Segundo da Hora et al. (2015) é preciso levar em consideração a sensibilidade possuída pela DEA em sua fronteira de eficiência, na qual uma grande quantidade de dados, se não tratados adequadamente, é capaz de influenciar no resultado final do modelo. Portanto, foi realizada uma análise de correlação entre critérios, sendo desconsiderados aqueles com forte incidência na relação (acima de 50\%). Em seguida, um julgamento da importância de cada critério por posição foi realizado pelos autores do trabalho, eliminando variáveis consideradas desnecessárias as funções de cada posição.

Tabela 1: Variáveis de avaliação por posição de jogador. AT: Atacante. G: Goleiro. Dir.: Direção de preferência. L: Lateral. MA: Meia atacante. MC: Meia central. ML: Meia Lateral. P: Ponta. Z: Zagueiro.

\begin{tabular}{|c|c|c|c|}
\hline Tipo & Variáveis & Dir. & Posições \\
\hline \multirow{3}{*}{ Input } & Minutos jogados & + & \multirow{3}{*}{ Todas } \\
\hline & Somatório $(€)$ de elencos da liga & - & \\
\hline & Valor $(€)$ de elenco do clube & + & \\
\hline \multirow{26}{*}{ Output } & Ameaças ofensivas interrompidas & + & $\mathrm{Z}$ \\
\hline & Cartões: Vermelhos & - & $\mathrm{Z}, \mathrm{MC}, \mathrm{ML}$ \\
\hline & Chutes: Fora da grande área & + & $\mathrm{L}, \mathrm{MC}, \mathrm{ML}$ \\
\hline & Cruzamentos: Bloqueados & + & $\mathrm{Z}, \mathrm{MC}, \mathrm{ML}$ \\
\hline & Cruzamentos: Certos & + & $\mathrm{L}, \mathrm{ML}, \mathrm{P}$ \\
\hline & Desarmes: Certos & + & $\mathrm{Z}, \mathrm{MC}, \mathrm{ML}$ \\
\hline & Domínios de bola errados & - & $\mathrm{L}, \mathrm{P}$ \\
\hline & Dribles: Certos & + & $\mathrm{L}, \mathrm{MC}, \mathrm{ML}, \mathrm{MA}, \mathrm{P}$ \\
\hline & Erros defensivos & - & $\mathrm{Z}, \mathrm{L}$ \\
\hline & Faltas: Cometidas & - & $\mathrm{Z}, \mathrm{L}, \mathrm{AT}$ \\
\hline & Faltas: Sofridas & + & Z, L, MC, MA, AT \\
\hline & Gols: Contra & - & $\mathrm{Z}, \mathrm{L}$ \\
\hline & Gols: Dentro da pequena área & + & $\mathrm{P}, \mathrm{AT}$ \\
\hline & Gols: Fora da grande área & + & $\mathrm{L}, \mathrm{MA}, \mathrm{P}, \mathrm{AT}$ \\
\hline & Gols: Fora da pequena área & + & $\mathrm{P}, \mathrm{AT}$ \\
\hline & Gols: Sofridos & - & $\mathrm{G}$ \\
\hline & Interceptações & + & $\mathrm{G}, \mathrm{P}$ \\
\hline & Jogada aéreas: Vencidas & + & $\mathrm{Z}, \mathrm{MC}, \mathrm{ML}, \mathrm{P}, \mathrm{AT}$ \\
\hline & Outros: Passes resultantes em finalização & + & MA, P \\
\hline & Passes curtos: Certos & + & $\mathrm{Z}, \mathrm{L}, \mathrm{MC}, \mathrm{ML}, \mathrm{MA}$ \\
\hline & Passes curtos: Resultantes em finalização & + & $\mathrm{MC}, \mathrm{AT}$ \\
\hline & Passes entre adversários: Assistência & + & $\mathrm{MC}$ \\
\hline & Passes entre adversários: Resultantes em finalização & + & ML, MA, AT \\
\hline & Passes longos: Certos & + & G, L, MA, P \\
\hline & Passes: Bloqueados & + & $\mathrm{L}$ \\
\hline & Perda de posse de bola & - & $\mathrm{Z}, \mathrm{MC}, \mathrm{AT}$ \\
\hline
\end{tabular}

Fonte: Elaborado pelos autores.

A Tabela 1 foi elaborada para descrever as variáveis de input e output utilizadas na pesquisa e, como mostrado pelo mesmo, foram utilizados um total de três inputs e 26 outputs. Observa-se que existem duas direções de preferência positivas e negativas, sendo estas ilustradas por seus sinais algébricos “+” e “_-", respectivamente. Para as variáveis de direção negativa, foi necessária uma modificação pelas restrições de não negatividade, descritas ao final das Equações 1 e 2, nas quais os pesos de input e output precisam ser iguais, ou maiores que zero. Tal ato foi feito de acordo com o procedimento proposto por Gomes et al. (2012), realizado conforme as seguintes etapas: (a) Multiplicar a variável por -1; (b) somar a variável o 
módulo do menor valor de seu conjunto. Desta forma todas as relações entre variáveis se mantiveram as mesmas, enquanto foram alteradas suas direções de preferência.

A respeito dos inputs, cabe aqui uma descrição dos mesmos. "Minutos jogados" é julgado como uma variável de entrada por ser determinante para a existência de qualquer outro tipo de desempenho de uma DMU, visto que não é possível que haja uma estatística de desempenho, sem que haja minutos jogados. "Somatório $(€)$ de elencos da liga" foi inserida como uma variável que diz respeito as equipes e jogadores que a DMU enfrenta durante sua temporada. Se o indivíduo se encontra em uma liga com alto valor de mercado, consequentemente, os clubes e jogadores que enfrenta também possuem tais valores elevados, o que resulta em uma dificuldade maior para a obtenção de seus outputs. De forma semelhante, "Valor (€) de elenco do clube" é uma variável que diz respeito ao clube pelo qual o jogador atuou durante a temporada. Dado que o futebol é um esporte coletivo, quando maior o valor de mercado do clube de um jogador, melhores são seus companheiros e, portanto, também maiores são suas chances de se destacar.

Os resultados da pesquisa apresentaram as quantidades de jogadores, avaliados por posição, junto ao número destes considerados eficientes. Em seguida, foram realizadas análises sobre o conjunto de jogadores eficientes por campeonato, realizando uma classificação de acordo com a média de jogadores eficientes por competição. Logo após foi analisada a eficiência por clube, observando-se a correlação entre o total de jogadores eficientes por clube $\mathrm{e}$ sua pontuação ao final da disputa de seu campeonato. Por fim, foi feita a análise de correlação entre valores de mercado dos jogadores e eficiência, assim como idade e eficiência, tanto de forma individual, quanto em conjunto, de acordo com suas posições.

\section{Resultados e Análises}

Através da análise de quartis as DMU's foram divididas em quatro classificações mediante a quantidade de tempo em campo que obtiveram durante a temporada. A Tabela 2 apresenta as quantidades de DMU's por quartil, assim como suas divisões:

Tabela 2: Divisão em quartis usadas na pesquisa.

\begin{tabular}{|c|c|c|c|}
\hline Q1 & Q2 & Q3 & Q4 \\
\hline $\begin{array}{c}\text { Minutos }{ }_{\text {DMU }} \leq \\
423\end{array}$ & $\begin{array}{c}424 \leq \text { Minutos } \\
1248\end{array}$ & $\begin{array}{c}1249 \leq \text { Minutos } \leq \\
2161\end{array}$ & $\begin{array}{c}\text { Minutos } \mathrm{DMU} \geq \\
2162\end{array}$ \\
\hline DMU's Q1 & DMU's Q2 & DMU's Q3 & DMU's Q4 \\
\hline 681 & 900 & 901 & 898 \\
\hline
\end{tabular}

Fonte: Elaborado pelos autores.

Foram considerados apenas as DMU's contidas em Q3 e Q4, o que representou um total de 1.799 indivíduos a serem avaliados. Posteriormente, estes foram separados de acordo com suas posições para terem suas eficiências calculadas, como demonstra a Tabela 3.

Observa-se que um total de 881 DMU's foram consideradas eficientes $(49 \%$ da população) de acordo com sua avaliação por variáveis específicas de sua função. Dentre as posições, destacam-se os Goleiros $(\mathrm{G})$, nos quais apenas $15,4 \%$ foram considerados eficientes. Este resultado pode ser relacionado a particularidade da posição, sendo uma função exclusiva, sem semelhança com as outras. A posição de Meia Lateral (ML) foi a que apresentou maior porcentagens de DMU's eficientes $(70,5 \%)$, tal comportamento possui relação com as diferentes funções que a posição pode desempenhar, podendo ser considerado tanto um defensor, quanto um atacante, variando conforme esquema tático do clube que representa.

Os resultados obtidos foram agrupados de acordo com os campeonatos nacionais disputados pelos jogadores, conforme Tabela 4. 
Tabela 3: Classificação de eficiência por posição. AT: Atacante. G: Goleiro. Dir.: Direção de preferência. L: Lateral. MA: Meia atacante. MC: Meia central. ML: Meia Lateral. P: Ponta. Z:

Zagueiro.

\begin{tabular}{|c|c|c|c|c|}
\hline Posição & DMU's & Outputs & Eficientes & \% \\
\hline ML & 95 & 9 & 67 & $70,5 \%$ \\
\hline AT & 206 & 9 & 112 & $54,4 \%$ \\
\hline P & 196 & 10 & 106 & $54,1 \%$ \\
\hline MA & 82 & 6 & 43 & $52,4 \%$ \\
\hline MC & 395 & 11 & 200 & $50,6 \%$ \\
\hline L & 321 & 12 & 162 & $50,5 \%$ \\
\hline Z & 361 & 11 & 169 & $46,8 \%$ \\
\hline G & 143 & 3 & 22 & $15,4 \%$ \\
\hline Total & $\mathbf{1 . 7 9 9}$ & - & $\mathbf{8 8 1}$ & $\mathbf{4 9 , 0 \%}$ \\
\hline
\end{tabular}

Fonte: Elaborado pelos autores.

Tabela 4: Classificação de eficiência por campeonato nacional. Camp.: Campeonato; Aval.: Avaliação; N.o: Número de jogadores; Ef.: Jogadores eficientes; AT: Atacante. G: Goleiro. Dir.: Direção de preferência. L: Lateral. MA: Meia atacante. MC: Meia central. ML: Meia Lateral. P:

Ponta. Z: Zagueiro.

\begin{tabular}{|c|c|c|c|c|c|c|c|c|c|c|}
\hline Car & Aval. & G & $\mathbf{L}$ & $\mathbf{Z}$ & MC & ML & MA & $\mathbf{P}$ & AT & \begin{tabular}{|l|} 
Total \\
\end{tabular} \\
\hline \multirow{3}{*}{ Inglês } & N. ${ }^{\circ}$ & 24 & 47 & 51 & 58 & 19 & 17 & 30 & 33 & 279 \\
\hline & Ef. & 7 & 29 & 34 & 39 & 15 & 14 & 23 & 25 & 186 \\
\hline & $\%$ & $29,2 \%$ & $61,7 \%$ & $66,7 \%$ & $67,2 \%$ & $78,9 \%$ & $82,4 \%$ & $76,7 \%$ & $75,8 \%$ & $66,7 \%$ \\
\hline \multirow{3}{*}{ Holandês } & N. ${ }^{\circ}$ & 19 & 42 & 48 & 60 & & & 34 & 22 & 227 \\
\hline & Ef. & 6 & 31 & 33 & 35 & . & 1 & 24 & 17 & 147 \\
\hline & $\%$ & $31,6 \%$ & $73,8 \%$ & $68,8 \%$ & $58,3 \%$ & $0 \%$ & $50,0 \%$ & $70,6 \%$ & $77,3 \%$ & $64,8 \%$ \\
\hline \multirow{3}{*}{ Alemão } & N. ${ }^{\circ}$ & 18 & 47 & 50 & 47 & 20 & 11 & 21 & 28 & 242 \\
\hline & Ef. & 0 & 19 & 24 & 25 & 16 & 5 & 7 & 16 & 112 \\
\hline & $\%$ & $0,0 \%$ & $40,4 \%$ & $48,0 \%$ & $53,2 \%$ & $80,0 \%$ & $45,5 \%$ & $33,3 \%$ & $57,1 \%$ & $46,3 \%$ \\
\hline \multirow{3}{*}{ Espanhol } & N. ${ }^{\circ}$ & 21 & 53 & 52 & 66 & 13 & 15 & 28 & 33 & 281 \\
\hline & Ef. & 4 & 25 & 19 & 29 & & 6 & 18 & 19 & 128 \\
\hline & $\%$ & $19,0 \%$ & $47,2 \%$ & $36,5 \%$ & $43,9 \%$ & $61,5 \%$ & $40,0 \%$ & $64,3 \%$ & $57,6 \%$ & $45,6 \%$ \\
\hline \multirow{3}{*}{ Francês } & N. ${ }^{\circ}$ & 23 & 54 & 57 & 61 & 10 & 14 & 29 & 32 & 280 \\
\hline & Ef. & 2 & 26 & 22 & 33 & ( & 7 & 14 & 12 & 125 \\
\hline & $\%$ & $8,7 \%$ & $48,1 \%$ & $38,6 \%$ & $54,1 \%$ & $90,0 \%$ & $50,0 \%$ & $48,3 \%$ & $37,5 \%$ & $44,6 \%$ \\
\hline \multirow{3}{*}{ Italiano } & N. ${ }^{\circ}$ & 20 & 36 & 61 & 69 & 23 & 7 & 15 & 39 & 270 \\
\hline & Ef. & 2 & 18 & 28 & 24 & 11 & 7 & 9 & 14 & 108 \\
\hline & $\%$ & $10,0 \%$ & $50,0 \%$ & $45,9 \%$ & $34,8 \%$ & $47,8 \%$ & $28,6 \%$ & $60,0 \%$ & $35,9 \%$ & $40,0 \%$ \\
\hline \multirow{3}{*}{ Turco } & $\mathrm{N}^{\circ}$ & 18 & 42 & 42 & 34 & 10 & 16 & 39 & 19 & 220 \\
\hline & Ef. & 1 & 14 & 9 & 15 & 8 & 8 & 11 & 9 & 75 \\
\hline & $\%$ & $5,6 \%$ & $33,3 \%$ & $21,4 \%$ & $44,1 \%$ & $80,0 \%$ & $50,0 \%$ & $28,2 \%$ & $47,4 \%$ & 34,19 \\
\hline
\end{tabular}

Fonte: Elaborado pelos autores.

Nota-se que o Campeonato Inglês foi o que deteve tanto o maior total, quanto porcentagem, de jogadores eficientes. Seguido pelo Holandês, Espanhol, Alemão, Francês, Italiano e Turco. Quanto a porcentagem de eficiência por posição, o Campeonato Holandês 
obteve o maior conjunto, sendo superior em quatro posições (Goleiros, Laterais, Zagueiros e Atacantes), seguido pelo Campeonato Inglês com três posições (Meia Central, Meia Atacante e Ponta) e o Francês com maior percentual de eficiência nos jogadores atuantes como Meias Laterais. A análise da porcentagem de eficiência torna-se válida pela particularidade que cada campeonato possui quanto a seus esquemas táticos. Por exemplo, em alguns campeonatos certas posições são mais comuns e outras mais raras, como ocorre com os Meias Laterais, inexistentes no Campeonato Holandês, mas comum nas outras competições. Outro fator que valida a análise é a quantidade diferente de jogos para cada campeonato. Enquanto nos Campeonatos Alemão, Holandês e Turco os clubes disputam 34 partidas, nos restantes são disputadas 38 .

Posterior a classificação de ligas, foi analisado o total de jogadores eficientes por clube de futebol. A Tabela 5 lista os resultados de 27 clubes, detentores dos cinco maiores totais de jogadores eficientes, assim como os campeonatos dos quais participaram e suas pontuações.

Tabela 5: Classificação de eficiência por clube.

\begin{tabular}{|c|c|c|c|c|}
\hline Clube & Campeonato & Pontuação & Classificação & Jogadores Eficientes \\
\hline Aston Villa & Inglês & 17 & $20^{\circ}$ & 14 \\
\hline Las Palmas & Espanhol & 44 & $11^{\circ}$ & 14 \\
\hline Everton & Inglês & 47 & $11^{\circ}$ & 13 \\
\hline Utrecht & Holandês & 53 & $5^{\circ}$ & 13 \\
\hline Ajaccio & Francês & 37 & $19^{\circ}$ & 13 \\
\hline Ingolstadt & Alemão & 40 & $11^{\circ}$ & 13 \\
\hline Cambuur & Holandês & 18 & $18^{\circ}$ & 12 \\
\hline Darmstadt & Alemão & 38 & $14^{\circ}$ & 12 \\
\hline Empoli & Italiano & 46 & $10^{\circ}$ & 12 \\
\hline Heracles & Holandês & 51 & $6^{\circ}$ & 12 \\
\hline Sporting Gijon & Espanhol & 39 & $17^{\circ}$ & 12 \\
\hline Swansea & Inglês & 47 & $12^{\circ}$ & 12 \\
\hline Vitesse & Holandês & 46 & $9^{\circ}$ & 12 \\
\hline Arsenal & Inglês & 71 & $2^{\circ}$ & 11 \\
\hline Bayern de Munique & Alemão & 88 & $1^{\circ}$ & 11 \\
\hline Bournemouth & Inglês & 42 & $16^{\circ}$ & 11 \\
\hline De Graafschap & Holandês & 23 & $17^{\circ}$ & 11 \\
\hline Excelsior & Holandês & 30 & $15^{\circ}$ & 11 \\
\hline Frosinone & Italiano & 31 & $19^{\circ}$ & 11 \\
\hline Leicester & Inglês & 81 & $1^{\circ}$ & 11 \\
\hline Liverpool & Inglês & 60 & $8^{\circ}$ & 11 \\
\hline Nijmegen & Holandês & 46 & $10^{\circ}$ & 11 \\
\hline Heerenveen & Holandês & 42 & $12^{\circ}$ & 11 \\
\hline Watford & Inglês & 45 & $13^{\circ}$ & 11 \\
\hline Southampton & Inglês & 63 & $6^{\circ}$ & 10 \\
\hline Stoke City & Inglês & 51 & $9^{\circ}$ & 10 \\
\hline West Bromwich Albion & Inglês & 43 & $14^{\circ}$ & 10 \\
\hline
\end{tabular}

Fonte: Elaborado pelos autores.

Analisando a Tabela 5 é possível identificar diversos clubes de reputação mundial como o Bayern de Munique, Arsenal e Liverpool, porém nota-se a presença de diversos clubes de menor expressão com pontuações baixas, como os clubes com maior quantidade de jogadores 
eficientes: Aston Villa e Las Palmas. O primeiro finalizou a temporada na última $\left(20^{\mathrm{a}}\right)$ colocação do Campeonato Inglês, enquanto o segundo na $11^{\mathrm{a}}$. No intuito de verificar a relação existente entre o desempenho individual dos jogadores de um clube (total da eficiência) com o desempenho do mesmo no campeonato disputado (medido pela pontuação), foi realizada uma análise de correlação entre as respectivas variáveis, ilustrado pela Figura 2.

Figura 2: Correlação entre total de jogadores eficientes e pontuação do clube.

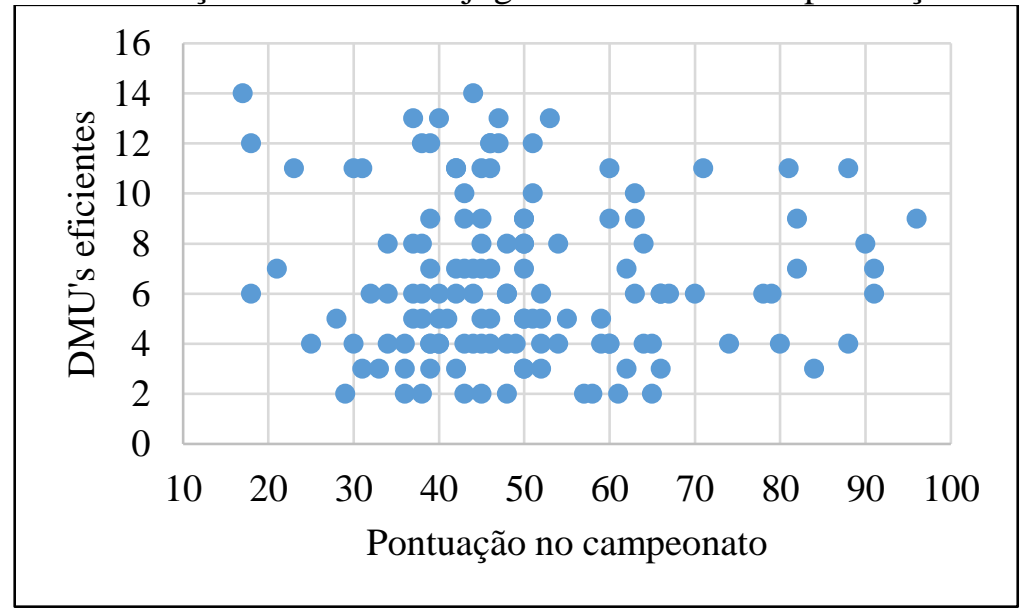

Fonte: Elaborado pelos autores.

Nota-se que não há nenhum tipo de relação entre as variáveis, seu valor de correlação (pela forma de Pearson) é de $-0,0562$, o que indica uma relação indiretamente proporcional, classificada como muito fraca, segundo Chowdhury et al. (2015), demonstrada na Tabela 6. Logo, não foram encontradas correlações entre as variáveis de desempenho coletivo e desempenho individual dos jogadores.

Tabela 6: Classificação adotada de valores de correlação pela forma Pearson.

\begin{tabular}{|c|c|}
\hline \multicolumn{2}{|c|}{ Intervalo dos valores de correlação } \\
\hline$|0,80|$ a $|1,00|$ & Muito forte \\
\hline$|0,60|$ a $|0,79|$ & Forte \\
\hline$|0,40|$ a $|0,59|$ & Moderada \\
\hline $0,20 \mid$ a $|0,39|$ & Fraca \\
\hline$|0,00|$ a $|0,19|$ & Muito fraca \\
\hline
\end{tabular}

Fonte: Adaptado de Chowdhury et al. (2015).

Outra análise de correlação foi realizada de acordo os jogadores e seus respectivos valores de mercado, tanto de forma conjunta (Figura 3), quanto individualmente de acordo com suas respectivas posições (Figura 4).

A correlação entre as variáveis representou um valor de 0,0148 , próxima de nula, sendo considerada muito fraca. Portanto, neste conjunto de dados, pode-se afirmar que não existe relação direta entre eficiência e valor de mercado dos jogadores.

A Figura 4 também analisa e demonstra as relações existentes entre as variáveis eficiência e valor de mercado, dividida conforme as oito posições desempenhadas.

Nota-se pela Figura 4 (a) que a posição de Goleiro foi a que obteve maior correlação entre eficiência e valor de mercado, resultando em um valor de -0,3293, porém ainda considerada uma correlação fraca. Outra posição que obteve nível de correlação fraca, foi a de Meias Armadores, mostrada na Figura 4 (f), com um valor de 0,2172. As demais posições obtiveram valores de correlação classificadas como muito fracas, com índices próximos a zero. 
Figura 3: Avaliação de eficiência e valor de mercado dos jogadores.

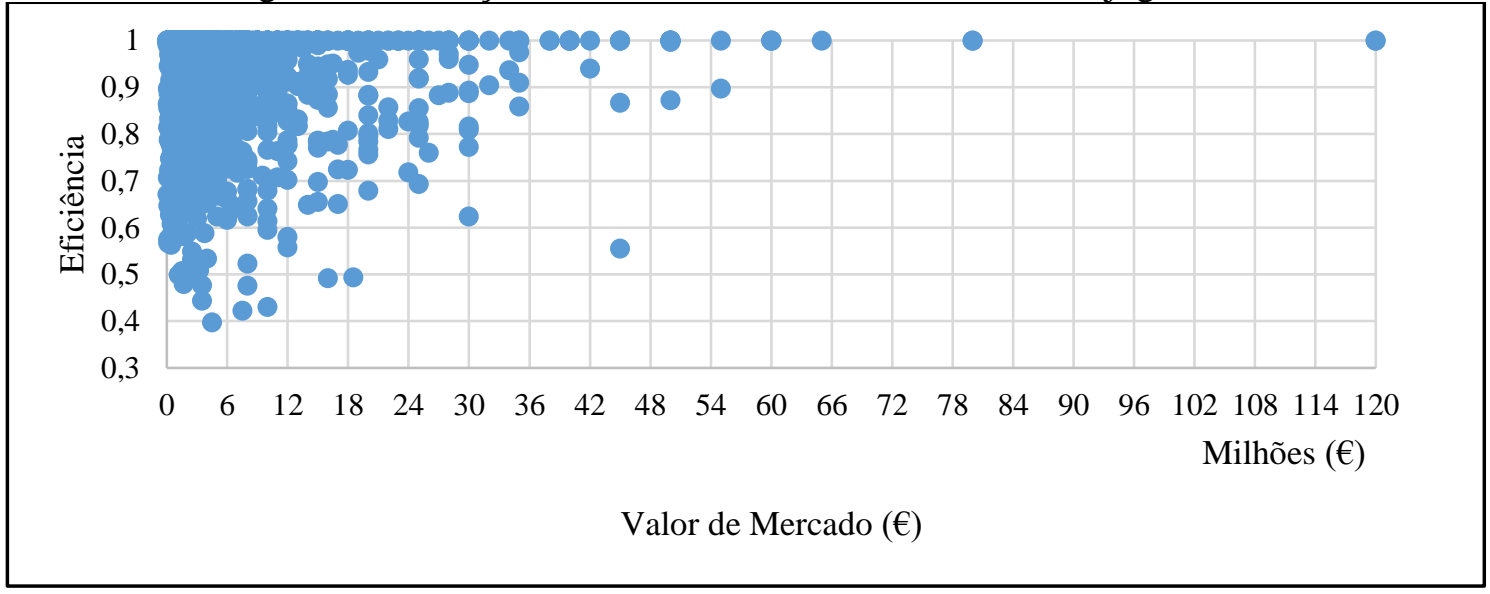

Fonte: Elaborado pelos autores.

Figura 4: Avaliação da eficiência e valor de mercado, conforme posições.
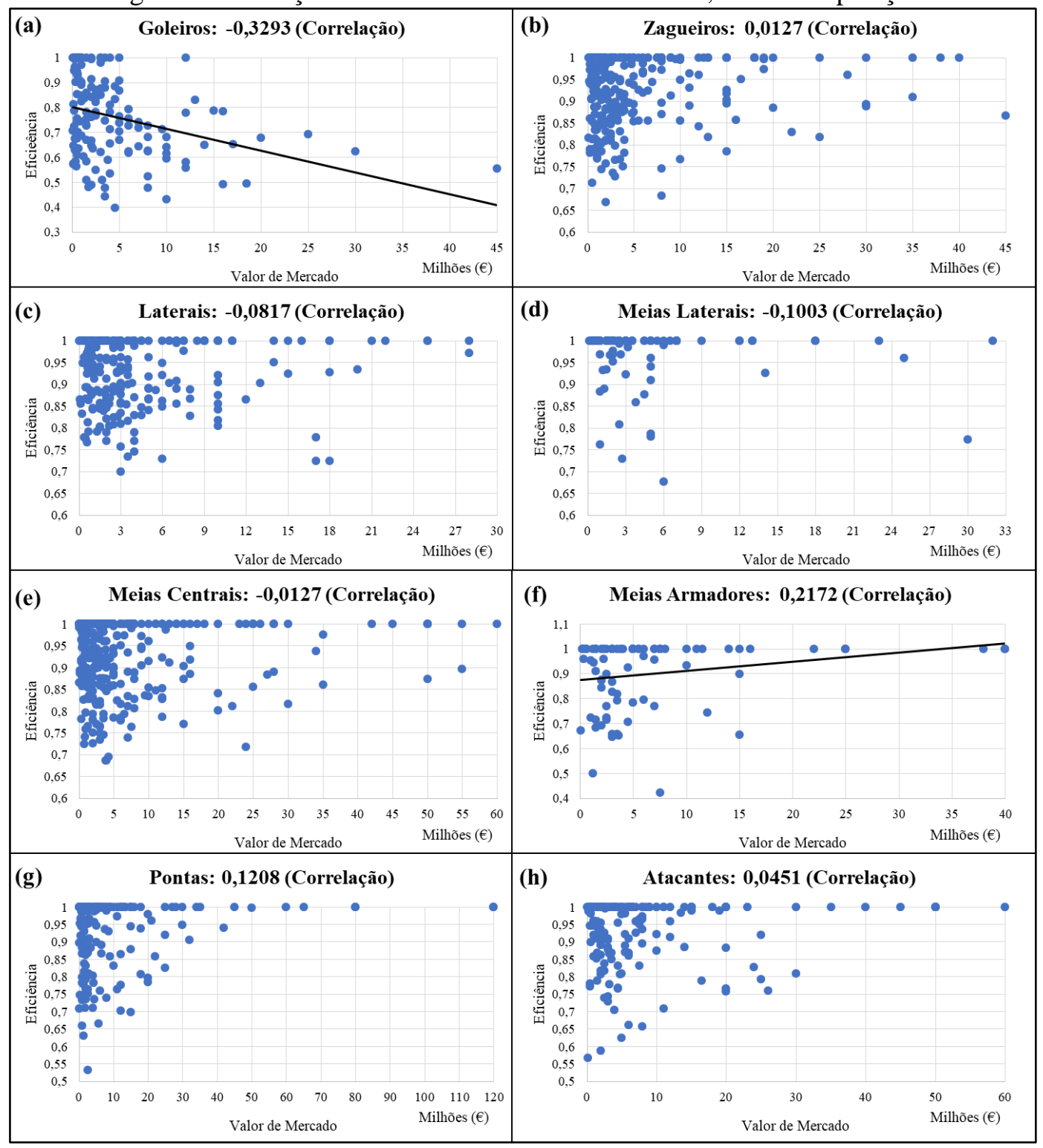

Fonte: Elaborado pelos autores. 
Outra hipótese levantada pela pesquisa foi a relação entre idade e eficiência dos jogadores. Os resultados entre tais variáveis são demonstrados pela Figura 5.

Figura 5: Relação entre eficiência e idade de jogadores.

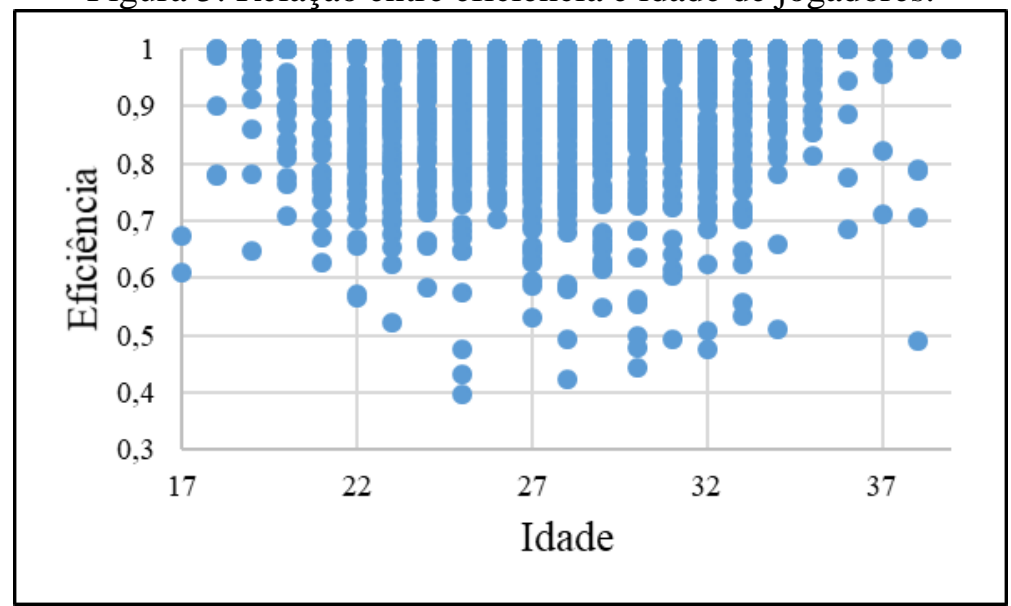

Fonte: Elaborado pelos autores.

O cálculo de correlação entre idade e eficiência também foi classificado como muito baixo $(-0,058)$. Em seguida, outro estudo foi feito entre as variáveis, visto que na relação idade e eficiência é possível que tanto o início quanto o final da carreira de um jogador representem intervalos menos eficientes. A fim de confirmar esta hipótese, foi elaborada a Tabela 7 na qual as idades foram divididas em três quartis, tendo cada extremo representando a parte inicial (Q1) e final (Q3) das carreiras dos jogadores.

Tabela 7: Análise de eficiência por idade. Q1: Quartil 1; Q2: Quartil 2; Q3: Quartil 3.

\begin{tabular}{|c|c|c|c|}
\hline AvaliaçãolQuartis & Q1 & Q2 & Q3 \\
\hline Jogadores Avaliados & 531 & 818 & 450 \\
\hline Eficientes & 278 & 403 & 200 \\
\hline$\%$ & $\mathbf{5 2 , 4 \%}$ & $49,3 \%$ & $44,4 \%$ \\
\hline
\end{tabular}

Fonte: Elaborado pelos autores.

Observando a Tabela 7, verifica-se que os indivíduos inseridos no Q1 (fase inicial da carreira) foram os detentores da maior média de eficiência, seguidos pelo segundo e terceiro quartis (Q2 e Q3, respectivamente). A mesma análise foi repetida de forma segmentada a cada uma das posições abordadas na pesquisa, a fim de identificar se há similaridade nos resultados apresentados pela Tabela 8 .

Os resultados indicados pela Tabela 8 , verificados por posição, não demonstraram estar alinhados ao geral demonstrado na Tabela 7. O primeiro quartil foi o de maior média de eficiência em apenas duas das oito posições (Lateral e Meia Central), enquanto que o segundo e terceiro quartis obtiveram maiores índices, cada um, em três funções dos indivíduos. Observa-se que não existiu nenhuma ligação entre os resultados, logo (de acordo com a amostragem estudada) não é possível afirmar que exista relação entre eficiência de jogadores e sua faixa etária. 
Tabela 8: Análise de eficiência por idade e posição. Q1: Quartil 1; Q2: Quartil 2; Q3: Quartil 3.

\begin{tabular}{|c|c|c|c|c|c|c|}
\hline Posições & \multicolumn{3}{|c|}{ Goleiro } & \multicolumn{3}{|c|}{ Zagueiro } \\
\hline Avaliação\Quartis & Q1 & Q2 & Q3 & Q1 & $\bar{Q} 2$ & Q3 \\
\hline Jogadores Avaliados & 42 & 56 & 45 & 81 & 193 & 87 \\
\hline Eficientes & 23 & 30 & 25 & 37 & 95 & 37 \\
\hline$\%$ & $54,8 \%$ & $53,6 \%$ & $55,6 \%$ & $45,7 \%$ & $49,2 \%$ & $42,5 \%$ \\
\hline Posições & \multicolumn{3}{|c|}{ Lateral } & \multicolumn{3}{|c|}{ Meia Lateral } \\
\hline AvaliaçãolQuartis & Q1 & Q2 & Q3 & Q1 & Q2 & Q3 \\
\hline Jogadores Avaliados & 72 & 180 & 69 & 17 & 61 & 17 \\
\hline Eficientes & 38 & 88 & 36 & 13 & 40 & 14 \\
\hline$\%$ & $52,8 \%$ & $48,9 \%$ & $52,2 \%$ & $76,5 \%$ & $65,6 \%$ & $82,4 \%$ \\
\hline Posições & \multicolumn{3}{|c|}{ Meia Central } & \multicolumn{3}{|c|}{ Meia Armador } \\
\hline AvaliaçãolQuartis & Q1 & Q2 & Q3 & Q1 & Q2 & Q3 \\
\hline Jogadores Avaliados & 93 & 205 & 97 & 17 & 46 & 19 \\
\hline Eficientes & 58 & 104 & 38 & 7 & 25 & 11 \\
\hline$\%$ & $62,4 \%$ & $50,7 \%$ & $39,2 \%$ & $41,2 \%$ & $54,3 \%$ & $57,9 \%$ \\
\hline Posições & \multicolumn{3}{|c|}{ Ponta } & \multicolumn{3}{|c|}{ Atacante } \\
\hline AvaliaçãolQuartis & Q1 & Q2 & Q3 & Q1 & Q2 & Q3 \\
\hline Jogadores Avaliados & 47 & 43 & 44 & 39 & 116 & 51 \\
\hline Eficientes & 24 & 25 & 18 & 20 & 64 & 28 \\
\hline$\%$ & $51,1 \%$ & $58,1 \%$ & $40,9 \%$ & $51,3 \%$ & $55,2 \%$ & $54,9 \%$ \\
\hline
\end{tabular}

Fonte: Elaborado pelos autores.

\section{Discussão e Trabalhos Relacionados}

Foram analisados diversos artigos de periódicos relacionados ao presente trabalho, nos quais houveram estudos com maior e menor relação, de acordo com o que foi realizado. Dentre os que apresentaram maior relação, destacam-se o trabalho de Tiedemann et al. (2011), o qual também analisou o desempenho de jogadores com DEA de acordo com suas posições, assim como foi realizado neste estudo, porém os autores utilizaram dados da liga alemã de 2002/03 a 2008/09, tendo apresentado uma relação positiva entre o score médio de eficiência de um jogador de um time e o ranking na tabela da liga no final da temporada. Um ponto interessante é que o presente trabalho utilizou dados de jogadores e clubes participantes dos campeonatos nacionais da Alemanha, Espanha, França, Holanda, Inglaterra, Itália e Turquia, referentes a temporada de 2015/2016, com uma base de dados considerada bastante extensa em comparação aos outros trabalhos relacionados. Já o trabalho de Caballero Fernández et al. (2012) também analisou a eficiência de jogadores com DEA, porém da Liga Espanhola de Futebol na temporada de 2009/2010, tendo a amostra de jogadores dividida em três grupos, de acordo com a posição. Os trabalhos mencionados possuem forte relação com esta pesquisa, no que diz respeito a seus objetivos. Porém, foram limitados a apenas uma liga nacional. Suas divisões quanto a posições de jogadores também foram menores que as realizadas nesta pesquisa. Enquanto em ambos os trabalhos, foram consideradas apenas três posições, ou funções dos jogadores, neste trabalho foram consideradas nove segmentações, as quais foram avaliadas de forma independente, apenas de acordo com as estatísticas consideradas relevantes para as mesmas, de acordo com a análise de correlação realizada anteriormente.

Por fim, ainda como um estudo fortemente associado a presente pesquisa, Añon et al. (2017) utilizaram como objeto de estudo os 23 jogadores classificados como melhores do mundo pela FIFA (Federação Internacional de Futebol Associação), os quais tiveram suas respectivas eficiências avaliadas para, em seguida, terem seus rankings de eficiência 
comparados a suas classificações atribuídas pela federação. Como input foi empregada a razão entre: valores de salário dos atletas e montante gasto em suas transferências, pelo número de temporadas que o jogador disputou por seu clube. Valendo-se de quatro outputs, (i) soma de tempo de jogos, (ii) porcentagem de pontos obtidos, (iii) saldo de gols das partidas disputadas pelo jogador, em 2015 e (iv) diferença entre valor de mercado do jogador quando chegou ao clube até seu valor no ano de 2015. Os resultados apontaram uma grande diferença entre as classificações de DEA e da FIFA, onde o considerado melhor jogador pela FIFA (Messi) foi ranqueado apenas na $4^{\mathrm{a}}$ posição pela DEA, enquanto o melhor classificado pela DEA (Thomas Müller), não estava entre os três melhores da federação esportiva. O estudo ainda apontou que nenhum dos três jogadores finalistas ao prêmio de melhor do mundo (Messi, Cristiano Ronaldo e Neymar) foram classificados como eficientes. Em tempo, tal como na presente pesquisa, foi identificado pelos autores que os jogadores mais caros, não são os mais eficientes. Logo, verifica-se uma semelhança entre a pesquisa de Añon et al. (2017) quanto aos resultados encontrados, referentes à fraca relação entre valor dos jogadores e suas eficiências. Porém, diferem-se no corte temporal realizado, onde os referidos autores analisaram o ano de 2015 , em sua totalidade, enquanto esta pesquisa analisou a temporada 2015/2016, composta por, aproximadamente, a metade dos dois anos. Ainda é necessário ressaltar que, tal como visto nos demais trabalhos relacionados, a presente pesquisa utiliza um conjunto de dados mais significante, tanto no que se referem às DMU's, quanto aos inputs e outputs, que foram específicos de acordo com as funções de cada jogador.

Arabzad et al. (2013) selecionaram e classificaram os melhores jogadores da English Premier League no período de 2010-2011 através da utilização de DEA em duas fases, uma para identificar os melhores jogadores e um para classificar os jogadores selecionados. Sua diferenciação ao presente trabalho reside, principalmente, nos dados utilizados para seu objetivo, os quais foram extraídos do jogo de Pro Evolution Soccer 2011, enquanto a presente pesquisa baseia-se apenas em dados estatísticos reais apresentados pelos jogadores.

De menor relação ao presente trabalho, Dadelo et al. (2014) seguiram uma metodologia diferente, na qual os autores propuseram a utilização de um algoritmo do método TOPSIS, considerando algoritmos como este simples e claros, sendo facilmente aplicados na prática. Santín (2014) propôs um modelo de Análise Envoltória de Dados de Supereficiência, sendo os resultados são calculados e comparados com a DEA padrão a fim de formar o time mais eficiente e supereficiente. A análise foi realizada empiricamente nos melhores jogadores do Real Madrid, conhecidos como "lendas brancas", desde a assinatura de Luis Molowny até a partida de Raúl Gonzalez. De forma similar, Oukil e Govindaluri (2017) apresentam uma metodologia para classificação de jogadores de futebol, integrando DEA e média ponderada ordenada. Os dados utilizados foram dos jogadores dos clubes europeus da Premier League, filtrando por fatores de desempenho desejáveis e indesejáveis, de acordo com o decisor. A robustez do método também foi avaliada de acordo com diferentes níveis de otimismo do decisor.

Muitos outros trabalhos analisam a eficiência de clubes de futebol com DEA, como é o caso de García-Sánchez (2007) que aplicou a DEA em três estágios de comportamento econômico para a Liga Espanhola de Futebol. Já Guzmán e Morrow (2007) analisaram a eficiência de clubes da English Premier League com base nas informações financeiras como medida de desempenho. Para isso, a técnica não paramétrica de Malmquist foi utilizada, sendo derivado de DEA e Análise de Correlação Canônica (CCA), de forma a garantir que haja coesão das variáveis de input e output. Barros e Douvis (2009) utiliza a DEA em um comparativo entre clubes de futebol da Grécia e Portugal no período de 1999/2000 a 2002/2003, buscando elencar as melhores práticas que possam levar a um melhor desempenho. Boscá et al. (2009) também utilizaram a DEA para analisar a eficiência, porém para clubes de futebol italiano e espanhol em três temporadas, resultando que a liga espanhola foi mais competitiva que a liga italiana. Já Jardin (2009) avalia a eficiência de clubes franceses de futebol com a DEA no período de 2004 a 2007. De forma similar, Wendlassida Miningou e Vierstraete (2012) também analisaram a eficiência de clubes franceses com DEA, no período de 2002/03 a 2007/08, utilizando os dados 
da Liga 1 e 2, medindo a eficiência técnica e a eficiência de escala. Os resultados indicaram uma ineficiência por parte dos clubes franceses, em geral.

\section{Considerações Finais}

A presente pesquisa, mediante seu caráter exploratório, analisou a eficiência de 1.798 jogadores, através do modelo DEA CCR, orientado a output, de sete dos principais campeonatos europeus de futebol e, posteriormente, testou duas hipóteses sobre a relação da eficiência com valores de mercado e idade dos indivíduos. Das DMU's estudadas, 49\% foram consideradas eficientes pelo modelo. Quanto as hipóteses estudadas:

- H1: Quanto maior o valor de mercado, mais eficiente será o jogador;

As relações entre as mencionadas variáveis foram testadas através da correlação de Pearson, tendo apenas duas de oito posições obtendo a classificação de correlação "fraca", enquanto as restantes foram qualificadas como "muito fracas" segundo a classificação adotada.

- H2: Jogadores são mais eficientes na fase intermediária de suas carreiras;

A referida hipótese foi testada a partir da divisão dos dados em quartis, na qual o primeiro e terceiro representariam as fases iniciais e finais da carreira, enquanto o segundo representaria o intermediário. Tanto de forma conjunta, como individual, não foi possível identificar nenhum tipo de padrão nas relações entre as variáveis e as médias de jogadores classificados como eficientes pelo modelo.

O número de jogadores, clubes e ligas analisados, justificam a relevância desta pesquisa para o futebol e para a literatura, a qual conta com escassez de trabalhos que utilizem a DEA para avaliação da eficiência de jogadores, principalmente no que tange a uma segmentação tão ampla de posições, como mostrados nesta pesquisa (nove, ao todo). Os resultados alcançados indicam que é possível que jogadores, independentemente de seus valores de mercado e idade, sejam considerados eficientes em suas funções. Fato que se apresenta como útil a gestores do meio no que cerne a tomada de decisão, mais especificamente sobre contratações de novos jogadores para seus clubes.

No entanto, pelo estudo realizado, não foi possível identificar padrões que permitissem sugestões sobre a melhoria de eficiência de jogadores e clubes, para que possam ser devidamente implementadas no meio profissional do futebol. Portanto, recomenda-se para pesquisas futuras a inserção de uma análise sobre a eficiência dos clubes, em conjunto com jogadores, para que informações sobre eficiência coletiva e individual possam ser confrontadas, em busca da identificação de padrões e relações que indiquem ações estratégicas para os gestores do meio. Também se considera válida a análise sobre uma maior amostragem, envolvendo campeonatos de outros países, durante um maior conjunto de temporadas, levando em consideração jogos de copas nacionais e campeonatos intercontinentais, a fim de confrontar os resultados obtidos neste trabalho que se ateve a temporada 2015/2016.

Agradecimentos: Os autores agradecem ao apoio financeiro prestado pelo Instituto Federal de Educação, Ciência e Tecnologia Fluminense (IFF) durante a elaboração da pesquisa.

\section{Referências}

Adler, N., Friedman, L. e Sinuany-Stern, Z. Review of ranking methods in the data envelopment analysis context. European Journal of Operational Research, v. 140, n. 2, p. 249-265, 2002. 
Añon, I. C., Scaglia, A. e Torezzan, C. Análise envoltória de dados aplicada para avaliação de jogadores de futebol. In: Anais do XLIX Simpósio Brasileiro de Pesquisa Operacional, Blumenau-SC. SOBRAPO, 2017. p. 580-588.

Arabzad, S. M., Ghorbani, M. e Shahin, A. Ranking players by DEA the case of English Premier League. International Journal of Industrial and Systems Engineering, v. 15, n. 4, p. 443-461, 2013.

Barros, C. P. e Douvis, J. Comparative analysis of football efficiency among two small European countries: Portugal and Greece. International Journal of Sport Management and Marketing, v. 6, n. 2, p. 183-199, 2009.

Boscá, J. E., Liern, V., Martínez, A. e Sala, R. Increasing offensive or defensive efficiency? An analysis of Italian and Spanish football. Omega, v. 37, n. 1, p. 63-78, 2009.

Caballero Fernández, R., Gómez Núñez, T. e Sala Garrido, R. Analysis of the efficiency of Spanish Soccer League Players (2009/10) using the metafrontier approach. Estudios de Economía Aplicada, v. 30, n. 2, p. 565-578, 2012.

Charnes, A., Cooper, W. W. e Rhodes, E. Measuring the efficiency of decision making units. European Journal of Operational Research, v. 2, n. 6, p. 429-444, 1978.

Chowdhury, K. A., Debsarkar, A. e Chakrabarty, S. Novel methods for assessing urban air quality: Combined air and noise pollution approach. Journal of Atmospheric Pollution, v. 3, n. 1, p. $1-8,2015$.

Da Hora, A. L. B., Shimoda, E., Da Hora, H. R. M. e Costa, H. G. Análise da eficiência dos serviços de saneamento básico nos municípios do estado do Rio de Janeiro. Pesquisa Operacional para o Desenvolvimento, v. 7, n. 1, p. 55-81, 2015.

Dadelo, S., Turskis, Z., Zavadskas, E. K. e Dadeliene, R. Multi-criteria assessment and ranking system of sport team formation based on objective-measured values of criteria set. Expert Systems with Applications, v. 41, n. 14, p. 6106-6113, 2014.

El-Demerdash, B. E., El-Khodary, I. A. e Tharwat, A. A. Developing a stochastic input oriented data envelopment analysis (SIODEA) model. International Journal of Advanced Computer Science and Applications, v. 4, n. 4, p. 40-44, 2013.

El-Demerdash, B. E., El-Khodary, I. A., Tharwat, A. A. e Shaban, E. R. Performance evaluation of European football teams using data envelopment analysis. In: Proceedings of the 10th International Conference on Informatics and Systems, Giza, Egito. ACM, 2016. p. 325-326.

Espitia-Escuer, M. e García-Cebrián, L. I. Team improvement in the UEFA Champions League: an application of data envelopment analysis. International Journal of Sport Management and Marketing, v. 16, n. 3-6, p. 172-197, 2016.

García-Sánchez, I. M. Efficiency and effectiveness of Spanish football teams: a three-stageDEA approach. Central European Journal of Operations Research, v. 15, n. 1, p. 21-45, 2007.

Gil, A. C. Como elaborar projetos de pesquisa. 5a. Edição, São Paulo: Atlas, 2010.

Gomes, E. G., de Mello, J. C. C. B. S., Meza, L. A., da Silveira, J. Q., Neto, L. B. e de Abreu, U. G. P. Some remarks about negative efficiencies in DEA models. In: Holtzman, Y. (Ed.), 
Advanced Topics in Applied Operations Management, cap. 7, p. 113-132. London: InTech, 2012.

González-Gómez, F. e Picazo-Tadeo, A. J. Can we be satisfied with our football team? Evidence from Spanish professional football. Journal of Sports Economics, v. 11, n. 4, p. 418442, 2010.

Guzmán, I. e Morrow, S. Measuring efficiency and productivity in professional football teams: evidence from the English Premier League. Central European Journal of Operations Research, v. 15, n. 4, p. 309-328, 2007.

Haas, D. J. Productive efficiency of English football teams-a data envelopment analysis approach. Managerial and Decision Economics, v. 24, n. 5, p. 403-410, 2003.

Hu, Z.-H., Zhou, J.-X., Zhang, M.-J. e Zhao, Y. Methods for ranking college sports coaches based on data envelopment analysis and PageRank. Expert Systems, v. 32, n. 6, p. 652-673, 2015.

Jardin, M. Efficiency of French football clubs and its dynamics. Munich Personal RePEc Archive, Paper n 19828, 2009.

Kiani Mavi, R., Kiani Mavi, N. e Kiani, L. Ranking football teams with AHP and TOPSIS methods. International Journal of Decision Sciences, Risk and Management, v. 4, n. 1-2, p. 108126, 2012.

Oukil, A. e Govindaluri, S. M. A systematic approach for ranking football players within an integrated DEA-OWA framework. Managerial and Decision Economics, v. 38, n. 8, p. 11251136, 2017.

Périco, A. E., Rebelatto, D. A. D. N. e Santana, N. B. Bank efficiency: are the largest banks the most efficient? A DEA analysis. Gestão \& Produção, v. 15, n. 2, p. 421-431, 2008.

Pestana Barros, C., Assaf, A. e Sá-Earp, F. Brazilian Football League technical efficiency: A Simar and Wilson approach. Journal of Sports Economics, v. 11, n. 6, p. 641-651, 2010.

Petrović Djordjević, D., Vujošević, M. e Martić, M. Measuring efficiency of football teams by multi-stage DEA model. Tehnicki vjesnik-Technical Gazette, v. 22, n. 3, p. 763-770, 2015.

Pyatunin, A. V., Vishnyakova, A. B., Sherstneva, N. L., Mironova, S. P., Dneprov, S. A. e Grabozdin, Y. P. The economic efficiency of European football clubs-data envelopment analysis (DEA) approach. International Journal of Environmental and Science Education, v. 11, n. 15, p. 7515-7534, 2016.

Ribeiro, A. S. e Lima, F. Portuguese football league efficiency and players' wages. Applied Economics Letters, v. 19, n. 6, p. 599-602, 2012.

Rubem, A. P. S. e Brandão, L. C. Multiple criteria data envelopment analysis - An application to UEFA EURO 2012. Procedia Computer Science, v. 55, p. 186-195, 2015.

Ruggiero, J. Frontiers in Major League Baseball: nonparametric analysis of performance using data envelopment analysis. New York: Springer, 2011.139p. 
Santín, D. Measuring the technical efficiency of football legends: who were Real Madrid's alltime most efficient players? International Transactions in Operational Research, v. 21, n. 3, p. 439-452, 2014.

Sexton, T. R., Silkman, R. H. e Hogan, A. J. Data envelopment analysis: Critique and extensions. New Directions for Evaluation, v. 1986, n. 32, p. 73-105, 1986.

Silva, E. L. D. e Menezes, E. M. Metodologia da pesquisa e elaboração de dissertação. $4^{\mathrm{a}}$ ed. Florianópolis: UFSC, 2005. Disponível em: <http://tccbiblio.paginas.ufsc.br/files/2010/09/024_Metodologia_de_pesquisa_e_elaboracao_de _teses_e_dissertacoes1.pdf>. Acesso em: 14/07/2017.

Squawka. The home of Football, Transfer and Club news. 2016. Disponível em: <http://www.squawka.com/news/>. Acesso em: 16/08/2017.

Tiedemann, T., Francksen, T. e Latacz-Lohmann, U. Assessing the performance of German Bundesliga football players: a non-parametric metafrontier approach. Central European Journal of Operations Research, v. 19, n. 4, p. 571-587, 2011.

Transfermarkt. Football transfers, rumours, market values, news and statistics. 2016. Disponível em: <http://www.transfermarkt.com/>. Acesso em: 16/08/2017.

UEFA - Union of European Football Associations. Federações-membro - Rankings da UEFA Rankings das competições de clubes da UEFA. 2016. Disponível em: <http://pt.uefa.com/memberassociations/uefarankings/country/season=2016/index.html > .

Acesso em: 16/08/2017.

Villa, G. e Lozano, S. Assessing the scoring efficiency of a football match. European Journal of Operational Research, v. 255, n. 2, p. 559-569, 2016.

Wendlassida Miningou, É. e Vierstraete, V. Efficience des clubs Français de football des Ligues 1 et 2. Revue D'économie Politique, v. 122, n. 1, p. 37-66, 2012.

Whoscored. Football Statistic - Football Live Scores - WhoScored.com. 2016. Disponível em: <https://www.whoscored.com/>. Acesso em: 16/08/2017.

Zambom-Ferraresi, F., García-Cebrián, L. I. e Lera-López, F. Performance evaluation in the UEFA Champions League. Journal of Sports Economics, v. 18, n. 5, p. 448-470, 2017. 